\title{
Vital Sign Detection Using 60-GHz Doppler Radar System
}

\author{
Te-Yu Jason Kao and Jenshan Lin \\ University of Florida, Gainesville, FL, 32611, USA \\ Email: teyukao@ufl.edu, jenshan@ufl.edu
}

\begin{abstract}
Non-contact vital sign detection using 60-GHz radar offers various advantages such as higher sensitivity and smaller antennas compared to lower-frequency systems, however, the respiration amplitude comparable to wavelength causes strong non-linear phase modulation, and relatively small heartbeat amplitude results in detection difficulty. In this paper, theoretical analysis and simulation of $60-\mathrm{GHz}$ detection are provided to address these issues. Both shallow and deep breathings are tested in the experiments, and the detection technique monitoring both the fundamental and second harmonic of respiration is proposed. The phenomena explained in the work can be applied to many millimeter-wave Doppler radar applications where target displacement is comparable to or larger than the wavelength to ensure robust detection.
\end{abstract}

Index Terms - Doppler radar, frequency measurement, microwave sensor, medical signal detection.

\section{INTRODUCTION}

The advance in non-contact vital sign detection based on microwave Doppler radar has made it an attractive option for healthcare and rescue applications [1]-[3]. The simple system architecture implemented by commonly used circuit blocks usually leads to a low-power and costeffective solution. Recently the detection using radar frequency near $60-\mathrm{GHz}$ ISM band to improve sensitivity has drawn increasing research attention [4] [5]. In the Doppler radar, transmitted signal is reflected and phase modulated by the target displacement $\mathrm{x}(\mathrm{t})$, and the baseband output $\mathrm{B}(\mathrm{t})$ can be approximated as

$$
\mathrm{B}(\mathrm{t}) \approx \cos \left\{\frac{4 \pi \mathrm{x}(\mathrm{t})}{\lambda}+\phi\right\}
$$

where $\lambda$ is the wavelength of radar signal, and $\phi$ is the total residue phase accumulated in the propagation path. Under the condition of $\mathrm{x}(\mathrm{t})<<\lambda$, higher radar operating frequency increases the sensitivity to small displacement, and shrink in antenna physical size facilitates a more compact system for portable devices.

However, the radar frequency at $60 \mathrm{GHz}$ is far beyond the optimal carrier frequency for vital sign detection revealed in [6], as the respiration displacement is comparable to $\lambda$. Post signal processing such as phase unwrapping is mentioned in [7], and further theoretical analysis and new detection methods are needed to improve the detection accuracy. Human vital sign sensing is a special case of two-tone sinusoidal vibration analyzed
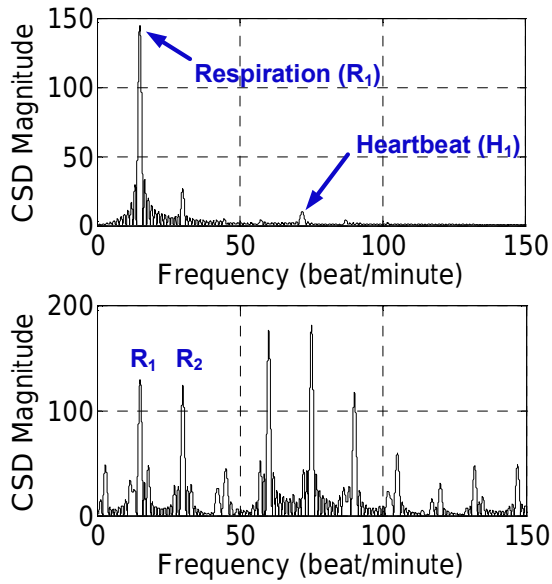

Fig. 1. Simulated vital sign detection using (a) $6 \mathrm{GHz}$ (b) 60 $\mathrm{GHz}$ radar as $\mathrm{m}_{\mathrm{r}}=2 \mathrm{~mm}$ and $\mathrm{m}_{\mathrm{h}}=0.2 \mathrm{~mm}$.

in [8], where the respiration amplitude $\left(\mathrm{m}_{\mathrm{r}} \approx 1-6 \mathrm{~mm}\right)$ is at least one order of magnitude larger than that of heartbeat $\left(\mathrm{m}_{\mathrm{h}} \approx 0.2 \mathrm{~mm}\right)$. The $\lambda$ at $60 \mathrm{GHz}(5 \mathrm{~mm})$ is no longer much greater than $\mathrm{m}_{\mathrm{r}}$ and the non-linear phase modulation results in strong harmonics and intermodulation. Figure 1 shows a simulated comparison between $6 \mathrm{GHz}$ and 60 $\mathrm{GHz}$ detection results after complex signal demodulation (CSD) [9]. In Fig. 1 (b), the relatively small heartbeat peak is overwhelmed by the harmonics of respiration even without the presence of system and environmental noise. In some cases, the fundamental respiration peak $\left(R_{1}\right)$ is too small to be distinguished and results in detection failure, which will be discussed in next section. This paper is organized as follows. The detection issues associated with $60-\mathrm{GHz}$ radar are simulated and explained in Section II, and techniques to improve respiration detection are provided. Section III shows the experiment results and discussion. Finally the conclusion is given in Section IV.

\section{DETECTION THEORY AND SimULATION}

Doppler radar shows alternating null and optimal detection points every $\lambda / 8$ as the detection distance varies $\phi$ [10]. In $60-\mathrm{GHz}$ system, the use of small $\lambda$ makes it impractical to avoid the null points by frequency or distance tuning, emphasizing the need to incorporate quadrature baseband outputs $\mathrm{B}_{1}(\mathrm{t})$ and $\mathrm{B}_{0}(\mathrm{t})$. As the chestwall displacement due to heartbeat and respiration is approximated by sinusoidal movement, $\mathrm{B}_{\mathrm{I}}(\mathrm{t})$ can be expressed as

U.S. Government work not protected by U.S. copyright 

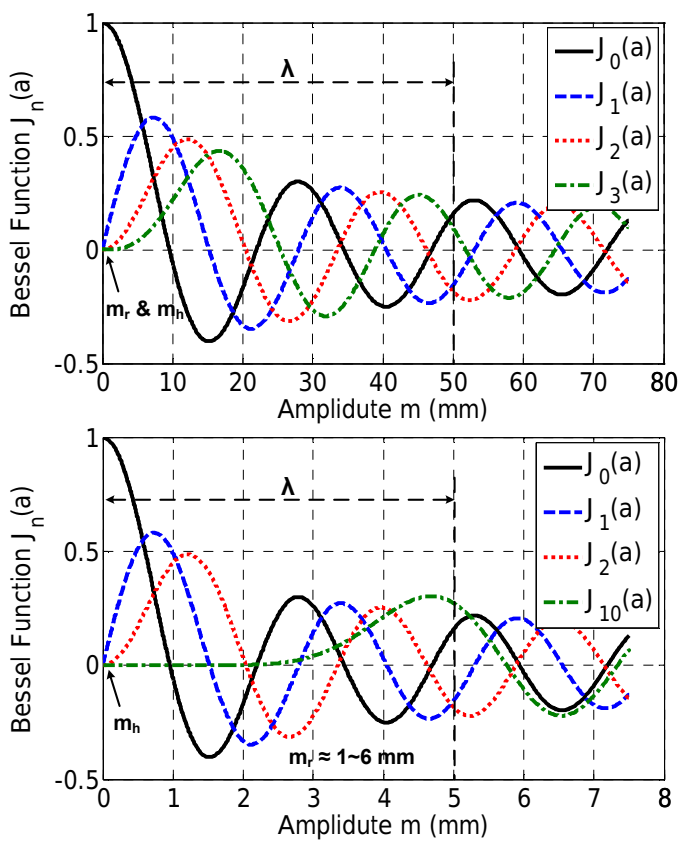

Fig. 2. $\quad J_{n}(a)=J_{n}(4 \pi m / \lambda)$ versus vibration amplitude at (a) 6 $\mathrm{GHz}$ (b) $60-\mathrm{GHz}$ radar frequency.

$$
\mathrm{B}_{\mathrm{I}}(\mathrm{t}) \approx \cos \left\{\frac{4 \pi[\mathrm{mr} \sin (2 \pi \mathrm{fr} t)+\mathrm{mh} \sin (2 \pi \mathrm{fht})]}{\lambda}+\phi\right\}
$$

where $f_{r}$ and $f_{h}$ are the respiration and heartbeat frequencies. In CSD process, complex signal $\mathrm{S}(\mathrm{t})$ is generated by combining I and Q baseband outputs [8]:

$$
\begin{aligned}
S(t) & =B_{I}(t)+j \cdot B_{Q}(t) \\
& =\exp \left\{j \cdot \frac{4 \pi\left[m_{r} \sin \left(2 \pi f_{r} t\right)+m_{h} \sin \left(2 \pi f_{h} t\right)\right]}{\lambda}+\phi\right\} \\
& =\sum_{k=-\infty}^{\infty} \sum_{p=-\infty}^{\infty} J_{k}\left(a_{r}\right) \cdot J_{p}(a h) \cdot \exp \left[j \cdot 2 \pi\left(k f_{r}+p f_{h}\right) t\right] \cdot e^{j \phi}
\end{aligned}
$$

where $\mathrm{a}_{\mathrm{r}}=4 \pi \mathrm{m}_{\mathrm{r}} / \lambda, \mathrm{a}_{\mathrm{h}}=4 \pi \mathrm{m}_{\mathrm{h}} / \lambda$, and $\mathrm{J}_{\mathrm{n}}$ is the $n$ th-order Bessel function of the first kind. The CSD spectrum is obtained by taking FFT of $\mathrm{S}(\mathrm{t})$, and the constant magnitude of $\exp (\mathrm{j} \phi)$ no longer affects the detection.

The heartbeat and respiration amplitudes with respective to wavelength determine the magnitude of harmonics and intermodulation. A peak at $\mathrm{x} \mathrm{Hz}$ is proportional to [7]:

$$
\mathrm{H}_{\mathrm{x}}=\left|\sum_{\mathrm{k}=-\infty}^{\infty} \sum_{\mathrm{p}=-\infty}^{\infty} \mathrm{J}_{\mathrm{k}}\left(\mathrm{ar}_{\mathrm{r}}\right) \cdot \mathrm{J}_{\mathrm{p}}\left(\mathrm{a}_{\mathrm{h}}\right)\right|
$$

where $k$ and $p$ are integers satisfying $\mathrm{k} \cdot \mathrm{f}_{\mathrm{r}}+\mathrm{p} \cdot \mathrm{f}_{\mathrm{h}}=\mathrm{x}$. For example, the fundamental respiration peak $\left(\mathrm{R}_{1}\right)$ located at $\mathrm{x}=\mathrm{f}_{\mathrm{r}}(\mathrm{k}=1$ and $\mathrm{p}=0)$ is represented by $\mathrm{J}_{1}\left(\mathrm{a}_{\mathrm{r}}\right) \cdot \mathrm{J}_{0}\left(\mathrm{a}_{\mathrm{h}}\right)$, and
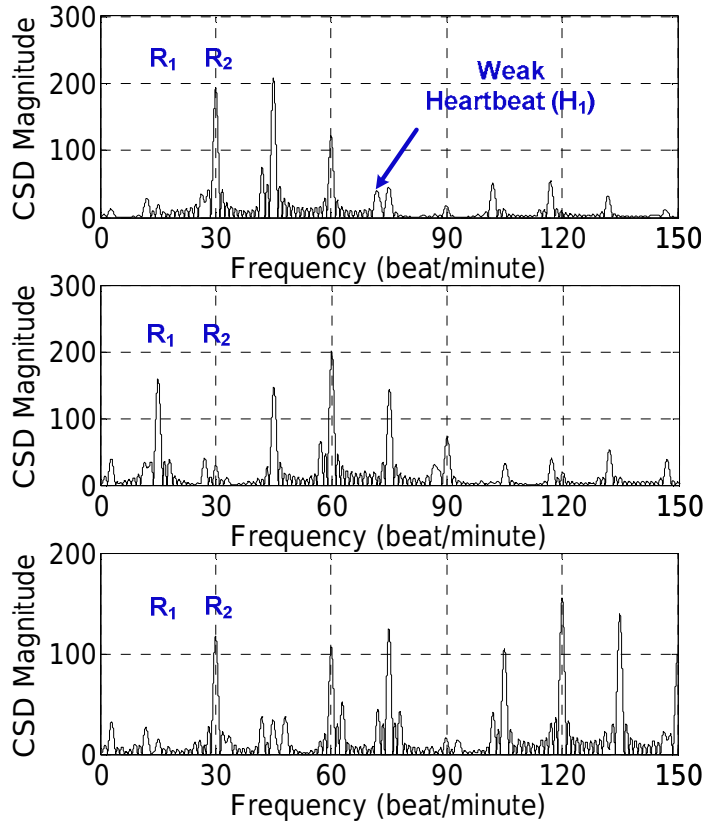

Fig. 3. Simulated vital sign spectrum at $60 \mathrm{GHz}$ as $\mathrm{m}_{\mathrm{r}}$ is (a) $1.5 \mathrm{~mm} \mathrm{(b)} 2.1 \mathrm{~mm} \mathrm{(c)} 4 \mathrm{~mm}$, and $\mathrm{f}$ is at 15 beat/minute. Heartbeat $\mathrm{m}_{\mathrm{h}}$ is fixed at $0.2 \mathrm{~mm}$ with $\mathrm{f}_{\mathrm{h}}=72$ beat $/$ minute.

the fundamental heartbeat peak $\left(\mathrm{H}_{1}\right)$ at $\mathrm{x}=\mathrm{f}_{\mathrm{h}}(\mathrm{k}=0$ and $\mathrm{p}$ $=1$ ) is determined by $\mathrm{J}_{0}\left(\mathrm{a}_{\mathrm{r}}\right) \cdot \mathrm{J}_{1}\left(\mathrm{a}_{\mathrm{h}}\right)$. As illustrated in the Bessel function plot in Fig. 2 (a), the small $m_{r}$ and $m_{h}$ compared to $\lambda$ at $6 \mathrm{GHz}(50 \mathrm{~mm})$ stay quite close to the origin on $\mathrm{x}$-axis. Ideally any harmonic and intermodulation $\mathrm{H}_{\mathrm{x}}$ consists of $\mathrm{J}_{\mathrm{n}}(\mathrm{a})$ with $|\mathrm{n}| \geq 3$ is small enough to be neglected, and this explains the spectrum in Fig. 1 (a) showing clear $\mathrm{R}_{1}$ and $\mathrm{H}_{1}$. It should be noted that Fig 2 . only plots $J_{n}(a)$ with positive $n$, and $J_{n}(a)$ with $n<0$ follows the symmetry of Bessel functions [8]:

$$
\mathrm{J}_{\mathrm{n}}(\mathrm{a})=\begin{gathered}
\mathrm{J}_{\mathrm{n}}(\mathrm{a}), \text { for even } \mathrm{n}<0 \\
-\mathrm{J}_{\mathrm{n}}(\mathrm{a}), \text { for odd } \mathrm{n}<0 .
\end{gathered}
$$

On the other hand, the detection scenario at $60 \mathrm{GHz}$ is quite different as presented in Fig. 2 (b). The heartbeat amplitude $m_{h}$ still stays near the origin on $x$-axis, but the high-order terms of $\mathrm{J}_{\mathrm{n}}\left(\mathrm{a}_{\mathrm{r}}\right)$ emerge as $\mathrm{m}_{\mathrm{r}}$ comparable to $\lambda$, resulting in a more complex spectrum. For example, as $m_{r}$ near $5 \mathrm{~mm}$, even $\mathrm{J}_{10}\left(\mathrm{a}_{\mathrm{r}}\right)$ is not negligible and thus generates a prominent peak of $\mathrm{R}_{10}$. The vital sign spectrum of various $m_{r}$ is simulated in Fig. 3 with the respiration rate at 15 beat $/$ minute. The follows discuss the $60-\mathrm{GHz}$ system's detection difficulties in terms of respiration and heartbeat, respectively.

\section{A. Respiration Detection at $60 \mathrm{GHz}$}

The fundamental and harmonics of respiration $\left(R_{1}, R_{2}\right.$ $R_{3}$, and etc) can be express as $J_{n}\left(a_{r}\right) \cdot J_{0}\left(a_{h}\right)$ with $n=1,2,3$, and etc. As the value of $J_{0}\left(a_{h}\right)$ is always close to unity, the respiration harmonics are generally larger than other 

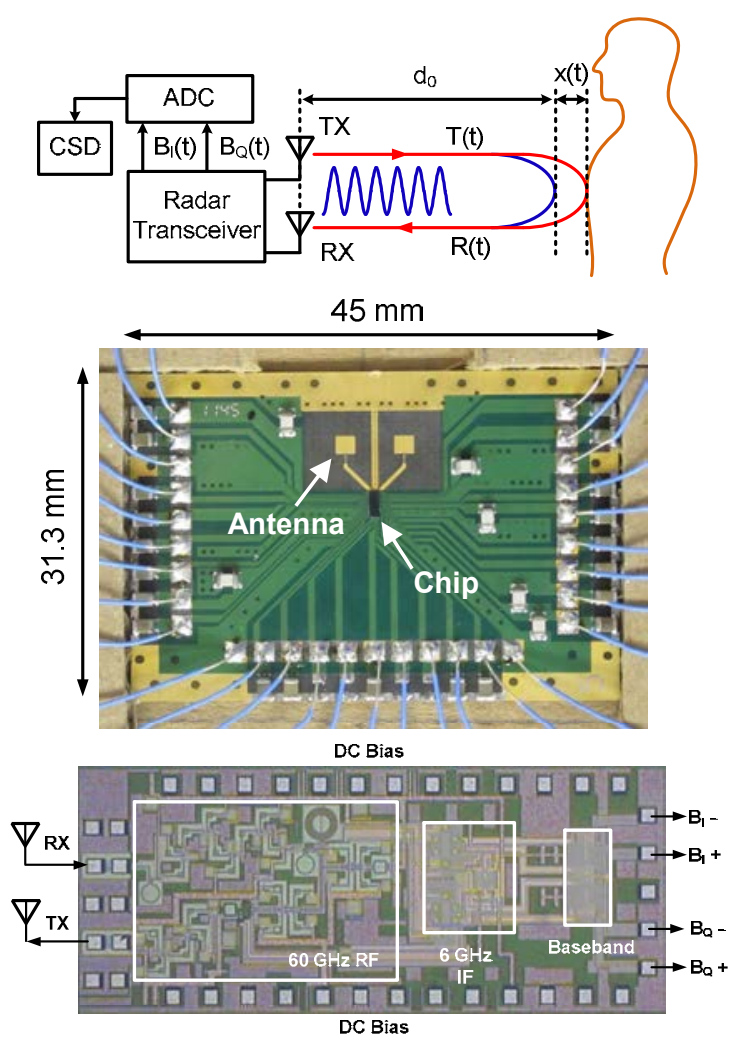

Fig. 4. (a) Vital sign detection experiment (b) Flip-chip integration with PCB patch antennas (c) CMOS radar transceiver chip.

intermodulation terms and can be easily identified on the spectrum in Fig. 3. However, the detection relying on $R_{1}$ is not robust since $\mathrm{J}_{1}\left(\mathrm{a}_{\mathrm{r}}\right)$ shows multiple zero-crossing points. As shown in Fig. 3 (a) and (c), $R_{1}$ vanishes as $m_{r}$ near 1.5 $\mathrm{mm}$ and $4 \mathrm{~mm}$. The respiration detection can be improved by monitoring $\mathrm{R}_{1}$ and $\mathrm{R}_{2}$ simultaneously. Observed from Fig. $2(b), J_{2}\left(a_{r}\right)$ approaches local maximum while $J_{1}\left(a_{r}\right)$ is near zero-crossing points, and vice versa. This implies theoretically the first prominent peak is either $\mathrm{R}_{1}$ or $\mathrm{R}_{2}$ as the frequency swept from low to high on the output spectrum, and the detection is able to covers all values of $\mathrm{m}_{\mathrm{r}}$. The frequency of other higher-order respiration harmonics can be used to distinguish between $R_{1}$ and $R_{2}$ by division, which will be demonstrated in Section III.

\section{B. Heartbeat Detection at $60 \mathrm{GHz}$}

The fundamental and harmonics of heartbeat $\left(\mathrm{H}_{1}, \mathrm{H}_{2^{\prime}}\right.$ $\mathrm{H}_{3}$, and etc) can be expressed as $\mathrm{J}_{0}\left(\mathrm{a}_{\mathrm{r}}\right) \cdot \mathrm{J}_{\mathrm{n}}\left(\mathrm{a}_{\mathrm{h}}\right)$ with $\mathrm{n}=1,2$, 3 , and etc. Normally the small value of $m_{h}$ near the origin on $\mathrm{x}$-axis in Fig. 2 (b) makes all $\mathrm{J}_{\mathrm{n}}\left(\mathrm{a}_{\mathrm{h}}\right)$ too small to be distinguished as respiration is present. In addition, the heartbeat peaks are also affected by respiration term $\mathrm{J}_{0}\left(\mathrm{a}_{\mathrm{r}}\right)$. In some rare cases as $\mathrm{J}_{0}\left(\mathrm{a}_{\mathrm{r}}\right)$ is near peak value $\left(\mathrm{m}_{\mathrm{r}} \approx 1.5\right.$ $\mathrm{mm})$ and the nearby respiration harmonics are weak, $\mathrm{H}_{1}$ can be seen on the spectrum as in Fig. 3 (a). If $\mathrm{J}_{0}\left(\mathrm{a}_{\mathrm{r}}\right)$ is near its zero-crossing points, it makes the already weak $\mathrm{H}_{1}$
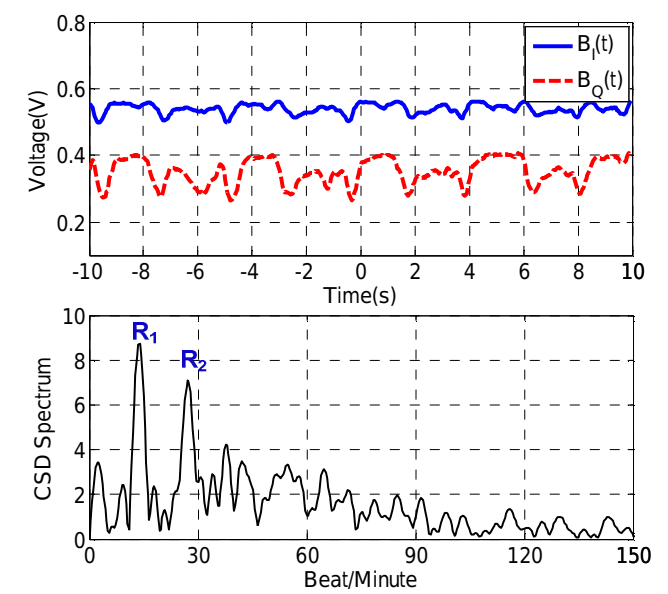

Fig. 5. (a) Time-domain (b) spectrum of vital sign detection results as the person breathes shallowly at $\mathrm{d}_{0}=0.3 \mathrm{~m}$.
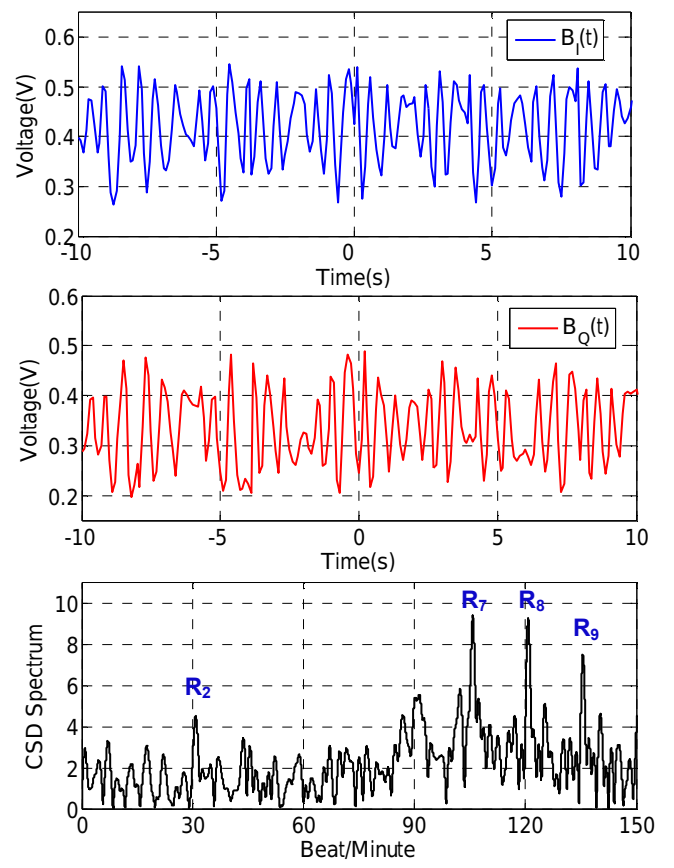

Fig. 6. (a) (b) Time-domain (c) spectrum of vital sign detection results as the person breathes deeply at $d_{0}=0.3 \mathrm{~m}$. The observation time is increased to $50 \mathrm{~s}$, but only $20 \mathrm{~s}$ is shown here for the comparison with Fig. 5.

more unlikely to be detected. Currently the heartbeat detection is obtained by holding the breath to avoid respiration harmonics on the output spectrum [4]. Detection from the back is an alternative way to reduce the interference from respiration.

\section{EXPERIMENT AND DISCUSSION}

The CMOS Doppler radar system operating at $55 \mathrm{GHz}$ [5] was used in the vital sign detection experiments shown in Fig. 4 (a). The radar transceiver chip was integrated with two PCB patch antennas by flip-chip process, and the system was pasted on an upright cardboard facing the 
target as displayed in Fig. 4 (b). Figure 4 (c) shows the radar transceiver chip which provides quadrature baseband outputs $\mathrm{B}_{\mathrm{I}}(\mathrm{t})$ and $\mathrm{B}_{\mathrm{O}}(\mathrm{t})$ that can be directly sampled for complex signal demodulation (CSD). As previously mentioned, the heartbeat can be detected by holding the breath, and I/Q channel outputs with CSD ensures the robust detection against distance $\left(d_{0}\right)$ [5].

Figure 5 shows the vital sign detection of a person sitting $0.3 \mathrm{~m}$ in front of the radar and breathing shallowly. $\mathrm{B}_{\mathrm{I}}(\mathrm{t})$ and $\mathrm{B}_{\mathrm{O}}(\mathrm{t})$ are displayed on an oscilloscope $\left(\mathrm{f}_{\mathrm{s}}=25\right.$ $\mathrm{Hz}$ ) and the observation time window is $20 \mathrm{~s}$. The results in Fig. 5 (b) shows the sallow breath mainly generates the fundamental ( $\mathrm{R}_{1}$ at 13.8 beat/minute) and second harmonic $\left(\mathrm{R}_{2}\right.$ at 27.2 beat/minute), and the detected respiration rate agrees with human counting. The higher-order harmonics are not prominent since $J_{n}\left(a_{r}\right) \cdot J_{0}\left(a_{h}\right)$ with $|n|>3$ are all small as predicted in Fig. 2 (b). From the plot $m_{r}$ can be estimated to be around $1 \mathrm{~mm}$ in this test.

As the target person breathing deeply at a rate of 15 beat/minute, Fig. 6 (a) and (b) shows the I and Q baseband outputs. The observation time window was increased to 50 $s$ and $\mathrm{f}_{\mathrm{s}}$ was $10 \mathrm{~Hz}$. It is noted that in $\mathrm{B}_{\mathrm{I}}(\mathrm{t})$ and $\mathrm{B}_{\mathrm{O}}(\mathrm{t})$, the modulated phase term $4 \pi \mathrm{x}(\mathrm{t}) / \lambda$ in (1) travels through multiples of $2 \pi$ due to large chest-wall movement $m_{r^{\prime}}$ resulting in much more complex waveforms compared to previous experiment. Figure 6 (c) shows the output CSD spectrum where the frequency resolution is improved by the longer observation time. As discussed in Section II, ideally the prominent peak at 30 beat/minute is either fundamental $\left(R_{1}\right)$ or second harmonic $\left(R_{2}\right)$ of respiration. Since the frequency of higher-order harmonics around 105 beat/minute $\left(\mathrm{R}_{\gamma}\right)$ and 135 beat/minute $\left(\mathrm{R}_{9}\right)$ are not dividable by 30 beat/minute, it is concluded that the peak around 30 beat/minute is $R_{2}$ and fundamental respiration frequency $\left(R_{1}\right)$ is at $f_{r}=15$ beat/minute. However from (4), theoretically there are expected to be more prominent harmonics such as $\mathrm{J}_{4}\left(\mathrm{a}_{\mathrm{r}}\right) \cdot \mathrm{J}_{0}\left(\mathrm{a}_{\mathrm{h}}\right)$ and $\mathrm{J}_{5}\left(\mathrm{a}_{\mathrm{r}}\right) \cdot \mathrm{J}_{0}\left(\mathrm{a}_{\mathrm{h}}\right)$ on the spectrum, which are not shown in the result of Fig. 6 (c). One of the possible reasons is that human respiration and heartbeat movements are not purely sinusoidal as modeled in (3) for simple analysis, resulting in the discrepancy of higher-order behavior.

\section{CONCLUSION}

Based on the analysis of $60-\mathrm{GHz}$ vital sign detection, the difficulty of respiration detection is mainly from the zero-crossing points of first-order Bessel function, which can be overcome by monitoring both fundamental and second harmonic peaks on the spectrum. For the already weak heartbeat peak, it is not only blocked by the respiration harmonics in the spectrum, but also affected by the zero-crossing points of zero-order Bessel function, making the simultaneous detection unlikely. Further baseband signal processing is needed to separate the respiration and heartbeat waveforms in time-domain before FFT is performed.

\section{ACKNOWLEDGEMENT}

For the $60-\mathrm{GHz}$ CMOS Doppler radar system used in the experiment, the authors wish to acknowledge the single-die bumping and flip-chip process sponsored by Mr. Terence Collier from CVInc, Richarson, Texas, USA. The authors are grateful to the $90 \mathrm{~nm}$ CMOS chip fabricated by United Microelectronics Corporation (UMC), Hsin-Chu, Taiwan R.O.C., and the microwave laminates provided by Rogers Corporation, Rogers, CT, USA.

\section{REFERENCES}

[1] K. M. Chen, Y. Huang, J. Zhang, and A. Norman, "Microwave life-detection systems for searching human subjects under earthquake rubble and behind barrier," in IEEE Trans. Biomed. Eng., vol. 47, pp.105-114, Jan 2000.

[2] A. D. Droitcour, O. Boric-Lubecke, V. M. Lubecke, J. Lin, and G. T. A. Kovac, "Range correlation and I/Q performance benefits in single-chip silicon Doppler radars for noncontact cardiopulmonary monitoring," in IEEE Trans. Microwave Theory \& Tech., vol. 52, no.3, pp. 838848, Mar. 2004.

[3] C. Li and J. Lin "Recent advances in Doppler radar sensors for pervasive healthcare monitoring," in IEEE Microwave Conference Proceedings (APMC), pp. 283-290, Dec. 2010.

[4] H. Chuang, H. Kuo, F. Lin, T. Huang, C. Kuo, and Y. Ou, "60-GHz millimeter-wave life detection system (MLDS) for noncontact human vital-signal monitoring," IEEE Sensors Journal, vol. 12, issue 3, pp. 602-609, Mar. 2012.

[5] T.-Y. J. Kao, A. Y.-K. Chen, Y. Yan, T. Shen, and J. Lin, "A flip-chip-packaged and fully-integrated $60 \mathrm{GHz}$ CMOS micro-radar sensor for heartbeat and mechanical vibration detections," IEEE Radio Freq. Integrated Circuits Symp., pp. 443-446, Jun. 2012.

[6] C Li and J. Lin, "Optimal carrier frequency of non-contact vital sign detectors," Proceedings of IEEE Radio and Wireless Symp., pp. 281-284, Jan. 2007.

[7] D. T. Petkie, C. Benton, and E. Bryan, "Millimeter wave radar for remote measurement of vital signs," IEEE Radar Conf., pp. 1-3, May 2009.

[8] Y. Yan, L. Cattafesta, C. Li, J. Lin, "Analysis of Detection Methods and Realization of a Real-time Monitoring RF Vibrometer," IEEE Trans. Microwave Theory \& Tech., vol. 59, no. 12, pp. 3556-3566, Dec 2011.

[9] C. Li and J. Lin, "Complex Signal Demodulation and Random Body Movement Cancellation Techniques for Non-contact Vital Sign Detection," IEEE MTT-S International Microwave Symp., pp. 567-570, June, 2008

[10] Y. Xiao, J. Lin, O. Boric-Lubecke, and V. M. Lubecke, "Frequency tuning technique for remote detection of heartbeat and respiration using low-power double-sideband transmission in Ka-band," IEEE Trans. Microwave Theory \& Tech., vol. 54, pp. 2023-2032, May 2006. 